\title{
ON THE OUT OF PHASE APPEARANCE OF LARGE-SCALE DIFFUSE MAGNETIC FIELD OF THE SUN WITH RESPECT TO SUNSPOTS
}

\author{
MAUSUMI DIKPATI ${ }^{1,2}$ and ARNAB RAI CHOUDHURI $I^{1,3}$ \\ 1 Department of Physics, Indian Institute of Science, Bangalore - 560012, India \\ 2 Indian Institute of Astrophysics, Bangalore - 560034, India \\ and \\ ${ }^{3}$ Kiepenheuer-Institut für Sonnenphysik, Schöneckstr. 6, D-79104 Freiburg, Germany
}

\begin{abstract}
.
We assume the large-scale diffuse magnetic field of the Sun to originate from the poloidal component of a dynamo operating at the base of the convection zone, whereas the sunspots are due to the toroidal component. The evolution of the poloidal component is studied to model the poleward migration of the diffuse field seen on the solar surface and the polar reversal at the time of sunspot maxima (Dikpati and Choudhuri 1994, 1995).
\end{abstract}

\section{Introduction}

Although the average latitude where the sunspots appear to migrate towards the equator, the large-scale weak diffuse magnetic fields of the Sun migrate poleward with the solar cycle (see Wang et al. 1989), the polar field reversing at the time of the sunspot maxima (first discovered by Babcock 1959). This is clearly seen in Figure 1, where the longitudinally averaged weak diffuse radial field and the number of sunspots are simultaneously plotted in a latitude-time diagram during the same time interval (May 1976 to December 1985).

The solar magnetic fields are presently believed to be generated in a thin layer at the base of the convection zone, where a dynamo wave propagates towards the equator (Choudhuri 1990; Parker 1993). If sunspots are produced from the toroidal magnetic field $\left(B_{\phi}\right)$, then their equatorward migration is easy to explain. We identify the weak, diffuse fields as the poloidal component $\left(\mathbf{B}_{p}\right)$ and study its evolution in the convection zone subject to turbulent diffusion and meridional circulation. We reproduce many features of the observational data reasonably well by adjusting the subsurface meridional flow suitably. 


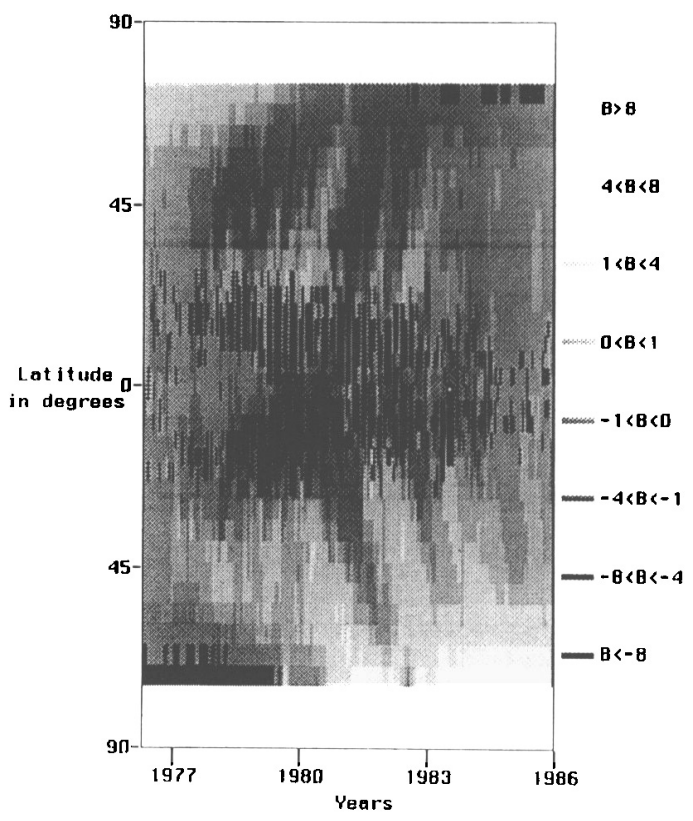

Fig. 1.

\section{Mathematical Formulation}

The evolution of magnetic fields inside the convection zone is governed by the induction equation, which reduces for an axisymmetric poloidal field $\left(\mathbf{B}=\nabla \times\left[A(x, z, t) \hat{\mathbf{e}}_{\phi}\right]\right)$ to a scalar equation

$$
\frac{\partial A}{\partial t}+s^{-1}\left(\mathbf{v}_{\mathbf{p}} \cdot \nabla\right)(s A)=\eta\left(\nabla^{2}-s^{-2}\right) A
$$

where $s=r \sin \theta$, with $\mathbf{v}_{\mathbf{p}}$ and $\eta$ representing the meridional flow and the diffusivity respectively. We solve (1) numerically within the convection zone with a specified meridional circulation $\mathbf{v}_{\mathbf{p}}$, which is equatorward at the bottom and poleward at the top (see Komm et al. 1993). An equatorward propagating dynamo wave is taken as the lower boundary condition. At the equator, the field lines of two hemispheres match smoothly, and at the pole, $A=0$ to keep the solution nonsingular. At the upper surface, the field lines smoothly match a potential field outside the Sun, which becomes radial at $2.5 R$ to take account of the stretching by the solar wind (see Altschuler and Newkirk 1969). 


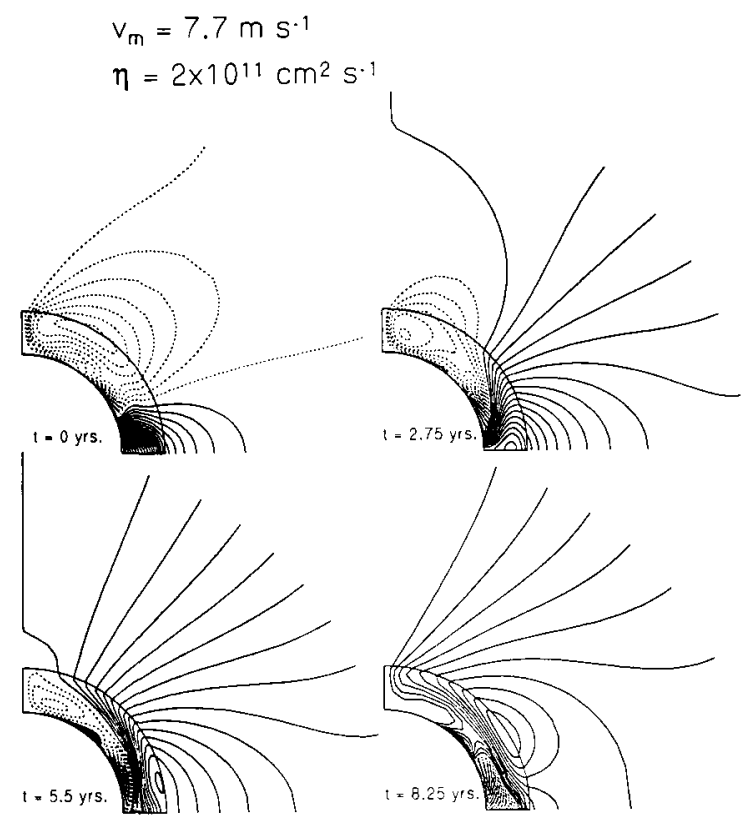

Fig. 2.

\section{Results and Conclusion}

Figure 2 shows the poloidal field lines at successive intervals of time. We see that the field lines at the bottom first move towards the equator where they join with their opposite hemisphere counterparts to form magnetic bubbles. These bubbles are pushed up due to the upwelling flow at the equator, and after reaching the surface, they are separated from their counterparts to drift towards the pole with the poleward surface flow. To compare our results with observations, we make a theoretical 'reverse butterfly diagram' from $B_{r}$ at the surface and superimpose on it a theoretical 'butterfly diagram' (Figure 3 ) obtained by introducing the appropriate phase lag between $B_{\phi}$ and $\mathbf{B}_{p}$ in the dynamo layer. We find that for a maximum surface flow of $6-8 \mathrm{~m} \mathrm{~s}^{-1}$ and a turbulent diffusion of $1-2 \times 10^{11} \mathrm{~cm}^{2} \mathrm{~s}^{-1}$, polar reversal occurs during the epoch of sunspot maximum (Dikpati and Choudhuri 1995).

Our model incorporates the full vector character of the magnetic field, whereas the previous flux transport models (see Wang et al. 1989) treated the magnetic field as a scalar on the solar surface and neglected all the subsurface physics. 


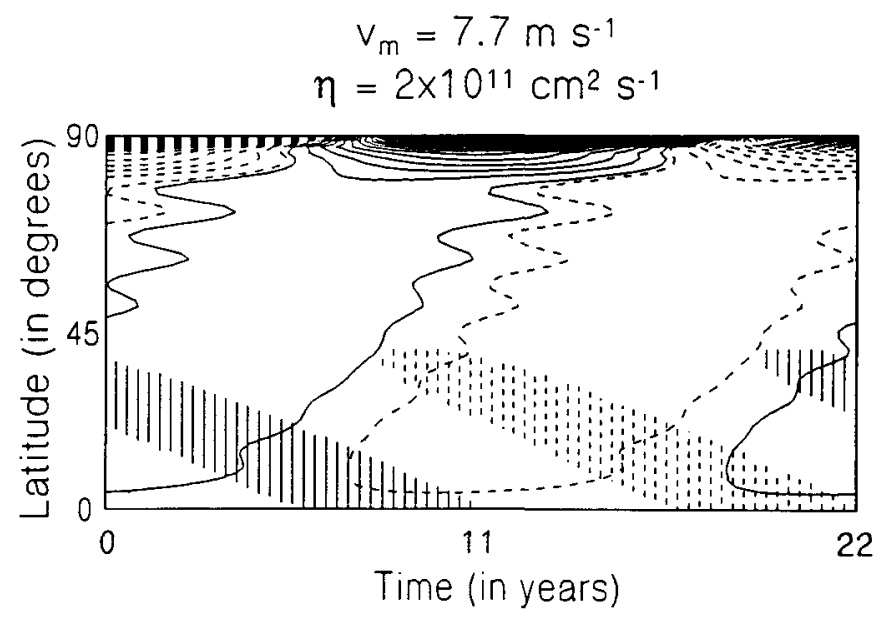

Fig. 3.

\section{Acknowledgements}

We thank Dr. R. Howard and Dr. Y.-M. Wang for supplying us the sunspot data and the data for longitudinally averaged photospheric fields respectively. M.D. thanks CSIR (India) for financial support. A.R.C.'s stay at the Kiepenheuer Institut was made possible by a Humboldt Fellowship.

\section{References}

Altschuler, M.D. and Newkirk, G.W., 1969, Solar Phys. 9, 131.

Babcock, H.D., 1959, Astrophys. J. 130, 364.

Choudhuri, A.R., 1990, Astrophys. J. 355, 733.

Dikpati, M. and Choudhuri, A.R., 1994, Aston. Astrophys., 291, 975.

Dikpati, M. and Choudhuri, A.R., 1995, Solar Phys., in press.

Komm, R.W., Howard, R.F., and Harvey, J.W., 1993, Solar Phys. , 147, 207.

Parker, E.N., 1993, Astrophys. J. 408, 707.

Wang, Y.-M., Nash, A.G., and Sheeley, N.R., 1989, Astrophys. J. 347, 529. 